\title{
Mitochondria from the left heart ventricles of both normotensive and spontaneously hypertensive rats oxidize externally added NADH mostly via a novel malate/oxaloacetate shuttle as reconstructed in vitro
}

\author{
ANNA ATLANTE ${ }^{1}$, TERESA M. SECCIA $^{2}$, LIDIA DE BARI $^{1}$, ERSILIA MARRA $^{1}$ and SALVATORE PASSARELLA ${ }^{3}$ \\ ${ }^{1}$ Istituto di Biomembrane e Bioenergetica, CNR, Bari; ${ }^{2}$ Dipartimento di Metodologia Clinica e Tecnologie Medico- \\ Chirurgiche, Università di Bari, Bari; ${ }^{3}$ Dipartimento di Scienze per la Salute, Università del Molise, Campobasso, Italy
}

Received January 12, 2006; Accepted March 3, 2006

\begin{abstract}
A substantial increase in NADH production, arising from accelerated glycolysis, occurs in cardiac hypertrophy and this raises the question of how the NADH is oxidised. We have addressed this problem by reconstructing appropriate mitochondrial shuttles in vitro, using mitochondria from the left ventricles of both normotensive and spontaneously hypertensive rats at 5 and 24 weeks of age as model systems for left ventricle hypertrophy and hypertrophy/hypertension respectively. We found that most NADH oxidation occurs via a novel malate/oxaloacetate shuttle, the activity of which increases with time and with the progression of hypertrophy and development of hypertension as judged by statistical ANOVA analysis. In contrast, $\alpha$-glycerol-phosphate and the malate/aspartate shuttles were shown to make only a minor contribution to NADH oxidation in a manner essentially independent of age and progression of hypertrophy/hypertension. The rate of malate transport in exchange with oxaloacetate proved to limit the rate of NADH oxidation via this malate/oxaloacetate shuttle.
\end{abstract}

\section{Introduction}

Normal cardiac function depends on the adequate delivery of oxygen and oxidisable substrates to mitochondria to generate sufficient ATP to meet the energy demands of the organs, including energy for both maintenance of structure and function of the myocardium and adequate reserve capacity to meet sudden surges in cardiac energy requirements (reviewed in ref. 1).

Correspondence to: Dr Salvatore Passarella, Dipartimento di Scienze per la Salute, Università del Molise, Via A. De Sanctis, 86100 Campobasso, Italy

E-mail: a.atlante@ibbe.cnr.it; passarel@unimol.it

Key words: hypertension, heart left ventricle, mitochondria, shuttle, $\mathrm{NADH}$, oxaloacetate
In general, the heart preferentially oxidizes fatty acids although glucose can also be used with the balance between utilization of lipids and carbohydrates correlating with their concentration in the arterial blood. In starvation, the energy demand in heart is also satisfied by ketone bodies $(2,3)$. Although severe cardiomyopathy and heart failure are accompanied by alterations in the balance of substrates used and of energy reserves, the myocardial metabolic changes were not thoroughly described in a heart with compensated hypertrophy (4) where, as a result of a significant decrease in fatty acid oxidation $(5,6)$, increased consumption of other substrates, notably of glucose, occurs $(7,8)$. Increased glycolitic flux in hypertrophied myocardium is important because ATP synthesis via glucose utilization may compensate for a decreased capacity for ATP synthesis by other pathways (9). How the glycolytic flux increases remains to be established.

In glycolysis, NADH is continuously produced in the glyceraldehyde-3-phosphate dehydrogenase reaction (EC 1.2.1.12). Since NADH cannot penetrate the internal mitochondrial space where Complex I of the respiratory chain, NADH-oxidoreductase (EC 1.6.5.3), is located, mitochondrial shuttles are required to oxidize cytosolic NADH. In these shuttles, NADH is oxidized in the cytosol by specific dehydrogenases, and the reduced metabolites so formed reach/enter the mitochondria. Mitochondrial enzymes then reoxidise the metabolites with concomitant reduction of mitochondrial cofactors. Finally, either directly in the case of the $\alpha$-glycerolphosphate shuttle or in a process that involves carrier-mediated transport, oxidised compounds are returned to the cytosol for further reaction with NADH (Fig. 1).

Studies with isolated mitochondria and with a perfused heart provide evidence that the malate/aspartate shuttle is sufficient to accommodate the highest rates of glycolytic NADH production under aerobic conditions (10). In this shuttle, both cytosolic and mitochondrial aspartate aminotransferases play a major role, even if, in the presence of aminooxyacetate (AOA), a transaminase inhibitor that enters mitochondria, only $20 \%$ inhibition of glucose oxidation was found (11). This poses the question as to whether other shuttles not involving transaminase reactions can contribute to glucose oxidation in the heart. In this regard, since oxaloacetate can enter rat heart 
mitochondria (RHM), causing the appearance of malate outside the mitochondria (12), the possibility exists that cytosolic $\mathrm{NADH}$ is oxidized in the heart via the malate/oxaloacetate shuttle as has already been shown to occur in brain, kidney, liver, plants and microorganisms (13-17).

In this report, we compare NADH oxidation by mitochondria isolated from spontaneously hypertensive (SHR) and normotensive Wistar-Kyoto (WKY) rats by adding to mitochondria both enzymes and substrates needed to reconstruct in vitro the malate/aspartate (MAL/ASP), $\alpha$-glycerol-phosphate $(\alpha \mathrm{GP})$ and malate/oxaloacetate (MAL/OAA) shuttles. We show, for the first time, the occurrence of the MAL/OAA shuttle in SHR and WKY mitochondria. MAL/OAA translocator regulates the rate of NADH oxidation. Of the three shuttles investigated, only the MAL/OAA shuttle showed increased activity, both in the initial and mature hypertensive state, using mitochondria isolated from 5- and 24-week old spontaneously hypertensive rats.

\section{Materials and methods}

Chemicals. All reagents were of the finest available grade with all solutions adjusted to $\mathrm{pH} 7.4$ by addition of either Tris or $\mathrm{HCl}$.

Animals. Two groups of male animals (Charles River, Calco, Italy) were used: spontaneously hypertensive (SHR) and Wistar-Kyoto (WKY) rats; the latter were used as a control. Thirty and 12 SHR or WKY rats at 5 and 24 weeks old, respectively, were used.

Blood pressure and left ventricular mass index. Systolic blood pressure (BP) was measured in conscious animals by using a tail-cuff plethismographic method (LE 5000 digital pressure meter, Letica, Barcelona, Spain). The values were taken at least three times and the mean value reported. Left ventricular mass index (LVMI) was calculated from the following formula: ventricle weight $(\mathrm{g}) /$ body weight $(\mathrm{g}) \times 10^{3}$ (18).

Isolation of mitochondria. Ten and 4 SHR and WKY male rats weighting 150-350 g (5- and 24-week old, respectively) were anesthetized with ether and sacrificed, and their hearts rapidly removed. The left ventricle was isolated after trimming both atria, right ventricle and connective tissue. Mitochondria $\left(\mathrm{RH}_{\mathrm{LV}} \mathrm{M}\right)$ were isolated from heart left ventricles, essentially according to Passarella et al (19), using a medium consisting of $0.25 \mathrm{M}$ sucrose, $20 \mathrm{mM}$ Tris- $\mathrm{HCl} \mathrm{pH} 7.25,1 \mathrm{mM}$ EGTA. The final mitochondrial pellet was suspended in the isolation medium to obtain $30-40 \mathrm{mg}$ protein/ml with mitochondrial protein measured according to Waddel and Hill (20). Since it is essential to use coupled mitochondria to investigate oxidative phosphorylation, each batch of mitochondria was tested for integrity and effectiveness in electron flow. Oxygen uptake measurements were carried out at $25^{\circ} \mathrm{C}$ in $2 \mathrm{ml}$ of a medium consisting of $100 \mathrm{mM} \mathrm{NaCl}, 10 \mathrm{mM} \mathrm{MgCl} 2,10 \mathrm{mM}$ sodiumphosphate buffer, $10 \mathrm{mM}$ Tris- $\mathrm{HCl} \mathrm{pH} \mathrm{7.4,} \mathrm{by} \mathrm{means} \mathrm{of} \mathrm{a}$ Gilson 5/6 oxygraph using a Clark electrode. Our mitochondrial preparations showed respiratory control ratios, i.e. the ratio between the rate of $\mathrm{O}_{2}$ consumption in state 3 (in the presence of ADP) and the rate in state 4 (in the absence of
$\mathrm{ADP}$ ), in the range of 3-5. $\mathrm{P} / \mathrm{O}$ ratios, i.e. the mole equivalents of phosphate esterified per oxygen consumed, were 3.0, 2.3 and 1.2 as obtained by using glutamate plus malate, succinate and ascorbate plus TMPD respectively as respiratory substrates.

Loading of mitochondria with aminooxyacetate. $\mathrm{RH}_{\mathrm{LV}} \mathrm{M}$ were suspended in $10 \mathrm{ml}$ of a medium containing $0.25 \mathrm{M}$ sucrose, $20 \mathrm{mM}$ Tris- $\mathrm{HCl}$ (pH 7.25), $1 \mathrm{mM}$ EGTA, $10 \mathrm{mM}$ aminooxyacetate (AOA) and $20 \mu \mathrm{g}$ rotenone. After $2 \mathrm{~min}$ at room temperature, the suspension was diluted to $50 \mathrm{ml}$ with icecold medium with AOA omitted. Mitochondria were collected by centrifugation and the resulting pellet was washed with AOA-free medium. A second sample of mitochondria was subjected to the same set of treatments but with AOA-free medium throughout. The complete inhibition of the intramitochondrial aspartate aminotransferase was checked essentially as in refs. 21 and therein.

Reconstruction of malate/oxaloacetate, glycerol-3-phosphate and malate/aspartate shuttles. MAL/OAA and $\alpha \mathrm{GP}$ shuttles were reconstructed at $25^{\circ} \mathrm{C}$, essentially as described $(13,22)$, and their activities measured using a Perkin-Elmer Lambda 19 spectrophotometer. In brief, $\mathrm{RH}_{\mathrm{LV}} \mathrm{M}$ suspended in $4 \mathrm{ml}$ of a standard medium containing $0.2 \mathrm{M}$ sucrose, $10 \mathrm{mM} \mathrm{KCl}$, $20 \mathrm{mM}$ HEPES-Tris, $\mathrm{pH} 7.2$ and $1 \mathrm{mM} \mathrm{MgCl}_{2}$, were placed in two cuvettes one of which was used as a reference. NADH $(0.2 \mathrm{mM})$ was added to the sample cuvette and the change in absorbance at $340 \mathrm{~nm}$ was monitored. Then, either malate dehydrogenase (MDH; EC 1.1.1.37) (2 e.u., i.e. $\mu$ moles of substrate transformed per minute at $25^{\circ} \mathrm{C}$ and $\mathrm{pH}$ 7.4) with $1 \mathrm{mM}$ MAL (in the case of MAL/OAA shuttle reconstruction) or $\alpha$-glycerol-3-phosphate dehydrogenase ( $\alpha \mathrm{GPDH}$; EC 1.1.1.8) (1 e.u.) with $1 \mathrm{mM} \alpha \mathrm{GP}$ (in the case of $\alpha \mathrm{GP}$ shuttle reconstruction) were added to both cuvettes and the change in absorbance was monitored at $340 \mathrm{~nm}$.

To continuously monitor the appearance of aspartate outside the mitochondria, a previously developed method (23) using an aspartate detecting system (ASP D.S.) was applied which consists of aspartate aminotransferase (AAT, 1 e.u.), the substrate pair $\alpha$-oxoglutarate $(\alpha \mathrm{OG}, 0.1 \mathrm{mM})$ and glutamate (GLU, $1 \mathrm{mM}$ ) and NADH (0.2 mM) plus MDH (2 e.u.). When studying the malate/aspartate shuttle, $\mathrm{RH}_{\mathrm{LV}} \mathrm{M}$ were added to $4 \mathrm{ml}$ of the standard medium and then two aliquots of the resulting suspension were put in two cuvettes, held in the photometer chamber. NADH plus MDH were added to one of them (the reference cuvette) and ASP D.S. was added to the other (the sample cuvette). The reaction was started by the simultaneous addition of malate to both cuvettes and oxidation of NADH arising from efflux of aspartate was measured spectrophotometrically. As the rate of NADH oxidation is measured as the difference between the rates measured in the sample cuvette and in the reference cuvette, this experimental protocol corrects for any possible appearance of OAA outside mitochondria due to reactions other than that catalysed by the externally added AAT; hence, the observed rate is that of the appearance of aspartate outside mitochondria.

Checks were always made to confirm that none of the compounds used in this study had any effect on the enzymes used to reveal metabolite appearance outside mitochondria, and also that the activities of the revealing enzymes did not 


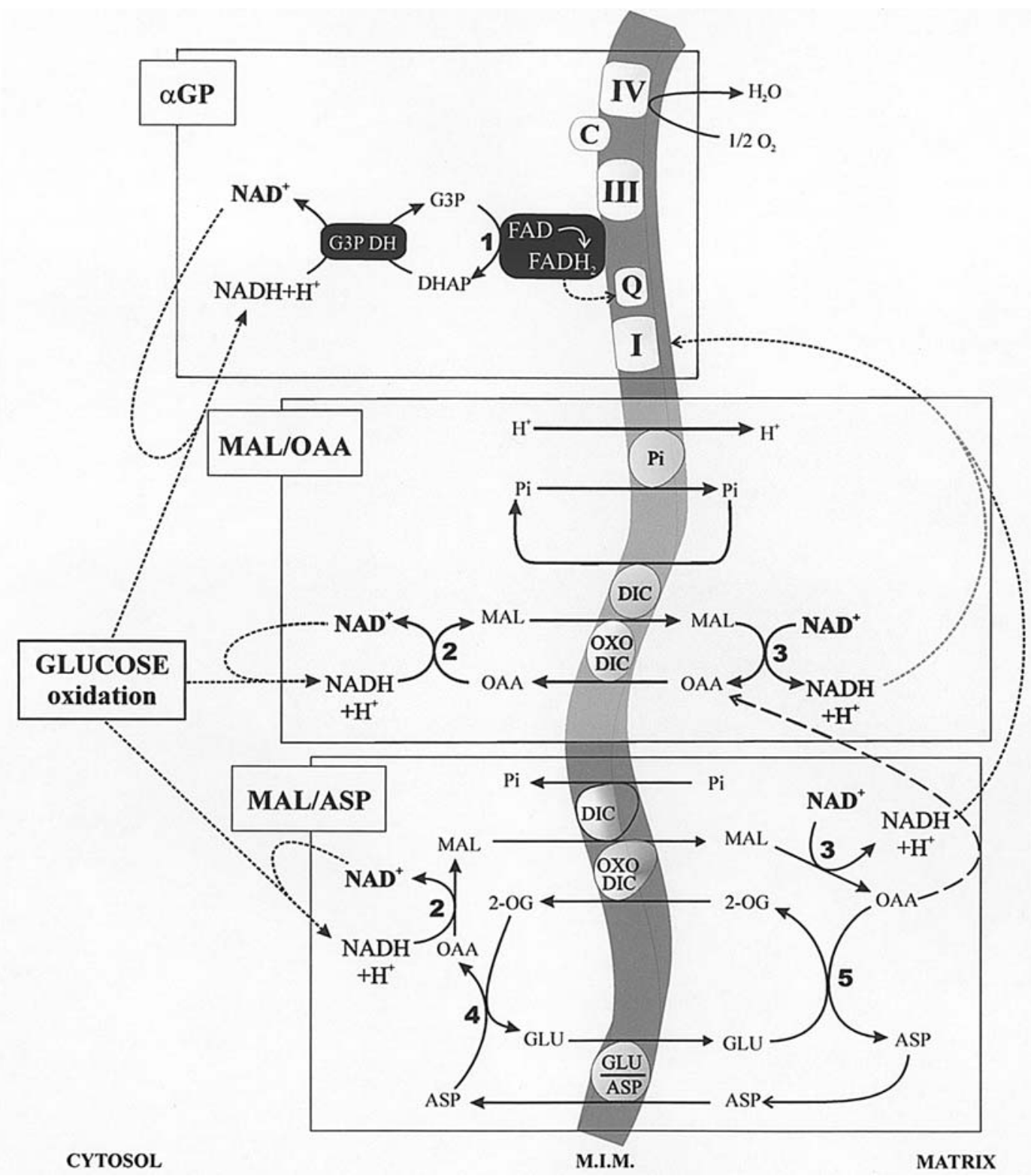

Figure 1. $\alpha \mathrm{GP}$, MAL/ASP and MAL/OAA shuttles in left ventricles of spontaneously hypertensive rats. MIM, mitochondrial inner membrane. Mitochondrial translocators: Pi, phosphate carrier; DIC, dicarboxylate translocator; OXODIC, oxodicarboxylate translocator; GLU/ASP, glutamate/aspartate translocator. Enzymes: 1, mitochondrial GPDH; 2 and 3, cytosolic and mitochondrial MDH respectively; 4 and 5, cytosolic and mitochondrial AAT respectively. For details see the text.

limit the overall reaction rate under the adopted experimental conditions. The rate of change of absorbance was obtained as the tangent to the initial part of the experimental curve in all cases and expressed as nmol of NADH oxidized per minute per $\mathrm{mg}$ of mitochondrial protein. The $\varepsilon_{340}$ value at $25^{\circ} \mathrm{C}$ for NADH was found to be $6.5 \mathrm{mM}^{-1} \mathrm{~cm}^{-1}$. NADPH, instead of $\mathrm{NADH}$, was used when the Triton experiment was carried out.

Measurement of aspartate aminotransferase activity in intact mitochondria. The method measures the intramitochondrial activity of aspartate aminotransferase and uses a Perkin-Elmer LS5 spectrofluorometer. The details of the assay method and the conditions under which it provides a true measurement of intramitochondrial enzyme activity have been reported (24). Briefly, $\mathrm{RH}_{\mathrm{LV}} \mathrm{M}$, suspended in $2 \mathrm{ml}$ of a standard medium in the presence of $2 \mu \mathrm{g}$ rotenone and $1 \mathrm{mM}$ sodium arsenite, were incubated with aspartate $(12 \mathrm{mM})$ and, after $1 \mathrm{~min}, \alpha$ oxoglutarate was added to a final concentration of $3 \mathrm{mM}$. The rate of decrease of fluorescence was then recorded and taken as a measurement of intramitochondrial AAT activity (24).

Statistical analysis and computing. All statistical analyses in this study were performed using SPSS software. The data were representative of at least four independent mitochondrial preparations (with comparable results), each one in triplicate, and are reported as the mean with standard deviation (SD). The statistical significance of the data was evaluated using one-way analysis of variance (ANOVA) followed by the posthoc Bonferroni test. $\mathrm{P}<0.05$ was considered as significant for all analyses. Experimental plots were obtained using Grafit (Erithacus software). 
Table I. Systolic blood pressure (BP) and left ventricular mass index (LVMI) in normotensive Wistar-Kyoto (WKY) and spontaneously hypertensive rats (SHR).

\begin{tabular}{llllll}
\hline & \multicolumn{2}{c}{ BP $(\mathrm{mmHg})$} & & \multicolumn{2}{c}{ LVMI } \\
\cline { 2 - 3 } \cline { 5 - 6 } Age & WKY & SHR & & WKY & SHR \\
\hline 5 weeks & $115 \pm 9$ & $125 \pm 10^{\mathrm{a}}$ & & $2.5 \pm 0.3$ & $3.1 \pm 0.3^{\mathrm{b}}$ \\
24 weeks & $138 \pm 15$ & $215 \pm 16^{\mathrm{a}}$ & & $2.3 \pm 0.2$ & $3.4 \pm 0.3^{\mathrm{a}}$
\end{tabular}

Mean $\pm \mathrm{SD}$. ${ }^{\mathrm{a} P}<0.001,{ }^{\mathrm{b}} \mathrm{P}<0.05$ vs same-aged WKY. Values are mean of five experiments performed in duplicate.

\section{Results}

Hypertrophy and hypertrophy-hypertension modelling. A significant difference in LVMI, but not in BP, was found when comparing 5-week old SHR ( $\left.\mathrm{SHR}_{5}\right)$ and WKY rats (Table I), in agreement with Das and Harris (25) and Engelmann et al (26), we assume that they represent a model in which any biochemical change might be related to ventricular hypertrophy (hypertrophic state). On the other hand, since biochemical alterations might occur as a result of the increase in both BP and LVMI (Table I) in 24-week-old rats $\left(\mathrm{SHR}_{24}\right)$, we consider them as exhibiting a hypertrophic-hypertensive state.

The oxidation of externally added NADH by left heart ventricle mitochondria in both hypertrophy and hypertension states: the reconstruction of the $\alpha G P, M A L / A S P$ and MAL/OAA shuttles. We isolated mitochondria from the left ventricles of SHR rats at 5 and 24 weeks of age $\left(\mathrm{SHR}_{5}-\mathrm{M}\right.$ and $\mathrm{SHR}_{24}-\mathrm{M}$ respectively) and WKY rats $\left(\mathrm{WKY}_{5}-\mathrm{M}\right.$ and $\left.\mathrm{WKY}_{24}-\mathrm{M}\right)$. Each of these mitochondrial preparations was used to reconstruct the $\alpha \mathrm{GP}, \mathrm{MAL} / \mathrm{ASP}$ and MAL/OAA shuttles and a typical set of experiments with $\mathrm{WKY}_{5}$-M is shown in Fig. 2. In all cases, the mitochondria were initially incubated with NADH
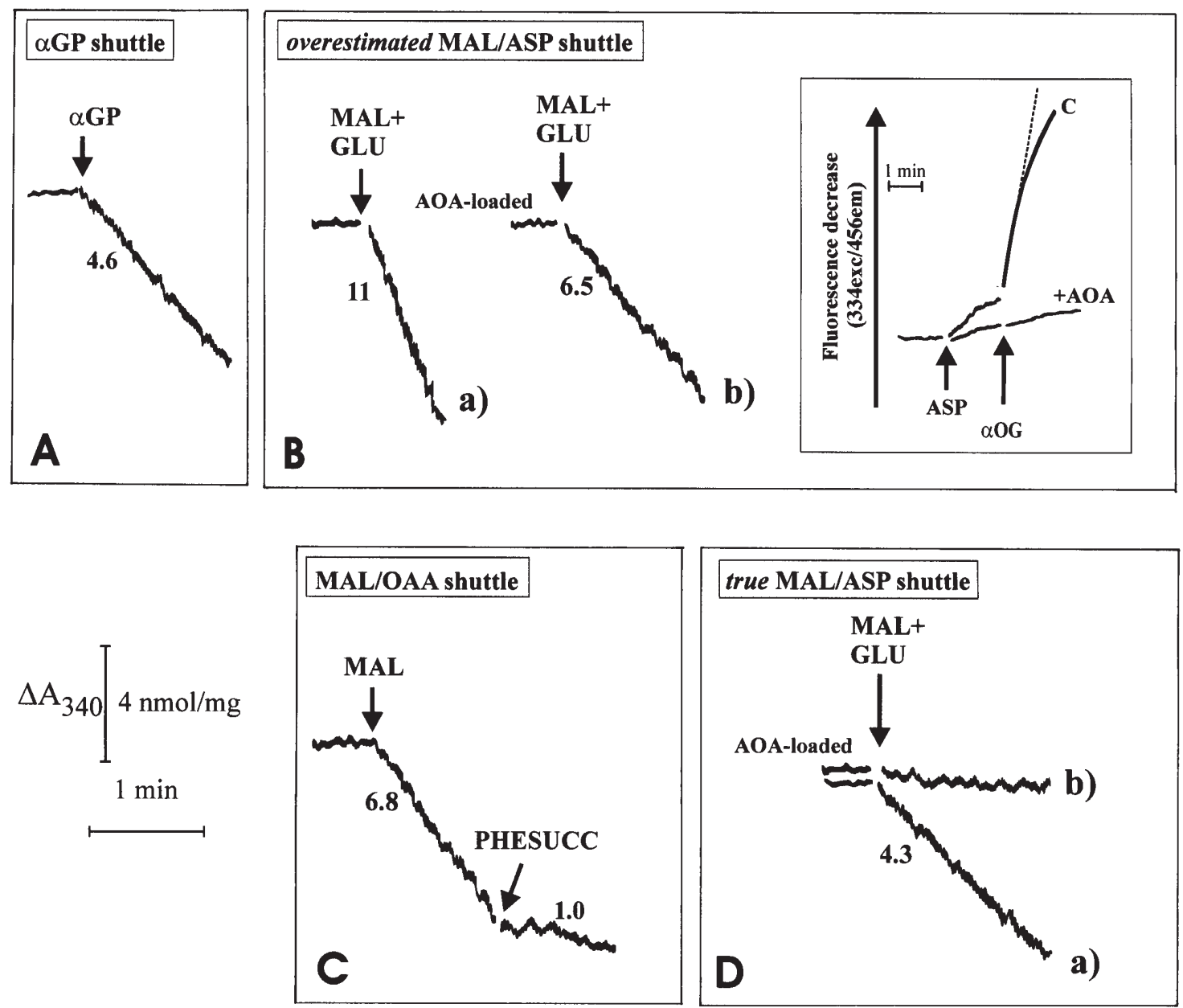

Figure 2. Oxidation of extramitochondrial NADH by $\mathrm{WKY}_{5}-\mathrm{M}$. Unloaded (A, Ba and C) or AOA-loaded-mitochondria ( $0.5 \mathrm{mg}$ protein) were incubated at $25^{\circ} \mathrm{C}$ in $2.0 \mathrm{ml}$ of standard medium, consisting of $0.2 \mathrm{M}$ sucrose, $10 \mathrm{mM} \mathrm{KCl}, 20 \mathrm{mM}$ HEPES-Tris, $\mathrm{pH} 7.2$ and $1 \mathrm{mM} \mathrm{MgCl}_{2}$, plus $0.2 \mathrm{mM} \mathrm{NADH}$ in the presence of $\alpha \mathrm{GPDH}$ (0.5 e.u.) (A), MDH (2 e.u.) (C), $\alpha$-oxoglutarate $(0.1 \mathrm{mM})$ plus $\mathrm{MDH}$ (2 e.u.) plus AAT (1 e.u.) (B and D) (for details see Materials and methods). Where indicated, additions were as follows: $1 \mathrm{mM} \alpha \mathrm{GP}, 1 \mathrm{mM} \mathrm{GLU}, 1 \mathrm{mM}$ MAL, $10 \mathrm{mM}$ PHESUCC. (B, inset) Mitochondrial AAT activity experiment: $\mathrm{RH}_{\mathrm{LV}} \mathrm{M}$, unloaded (C) or AOA-loaded-mitochondria (+ AOA), suspended in $2 \mathrm{ml}$ of standard medium in the presence of $2 \mu \mathrm{g}$ rotenone and $1 \mathrm{mM}$ sodium arsenite, were added with aspartate (ASP, $12 \mathrm{mM}$ ) and, after $1 \mathrm{~min}, \alpha$-oxoglutarate $(\alpha \mathrm{OG}, 3 \mathrm{mM})$. The rate of decrease of fluorescence $\left(\lambda_{\text {exc }} 334 \mathrm{~nm} /\right.$ $\lambda_{\mathrm{em}} 456 \mathrm{~nm}$ ) was then recorded and taken as a measurement of intramitochondrial AAT activity. Numbers along curves are rates of change in absorbance at $340 \mathrm{~nm}$ measured as tangents to the initial part of the progress curves and expressed as nmol NADH oxidised/min x mg mitochondrial protein. 
$(0.2 \mathrm{mM})$ and the absorbance at $340 \mathrm{~nm}$ was monitored for a short period of time to establish mitochondrial intactness; the constancy of absorbance at $340 \mathrm{~nm}$ demonstrated that the internal mitochondrial Complex I was inaccessible to NADH, i.e. the intactness of mitochondria. Then, the appropriate substrates and enzymes were added to reconstruct either the $\alpha \mathrm{GP}$ or the MAL/ASP shuttles and to establish the occurrence of the malate/OAA shuttle. In all cases, the operation of the shuttle was monitored continuously as a decrease with time of the NADH absorbance at $340 \mathrm{~nm}$ arising from metabolite interaction with (in the case of $\alpha \mathrm{GP}$ shuttle) or traffic across (in the case of the other two shuttles) the mitochondrial membrane as revealed using specific substrate detecting systems (Fig. 1).

In Fig. 2A $\alpha$ GPDH (1 e.u.) was added to mitochondria. The DHAP concentration in the extramitochondrial phase was negligible since no change of absorbance occurred. As a result of addition of $\alpha \mathrm{GP}(1 \mathrm{mM})$, a decrease in absorbance was observed at a rate equal to $4.6 \mathrm{nmol} \mathrm{NADH}$ oxidized/min $\mathrm{x} \mathrm{mg}$ mitochondrial protein. The explanation for this finding (Fig. 1) is that added $\alpha \mathrm{GP}$ is oxidized by mitochondria in the reaction catalyzed by mitochondrial $\alpha \mathrm{GPDH}(\mathrm{m} \alpha \mathrm{GPDH})$ located in the outer phase of the inner membrane (27) and the product, DHAP, in turn oxidizes NADH outside mitochondria in the reaction catalyzed by the added $\alpha \mathrm{GPDH}$.

Initial attempts to measure NADH oxidation as a result of the operation of the MAL/ASP shuttle were carried out essentially as described previously $(10,11,28)$. Mitochondria were incubated with the aspartate detecting system (ASP D.S.), consisting of $0.2 \mathrm{mM} \mathrm{NADH}, 0.1 \mathrm{mM} \alpha \mathrm{OG}, 0.5$ e.u. MDH plus 1 e.u. AAT. Then, malate plus glutamate $(1 \mathrm{mM}$ each) were added to the sample and the change in absorbance was measured (Fig. 2B). In this case, efflux of aspartate from the mitochondria is followed by its transamination, via AAT, to OAA which in turn is reduced to malate in a reaction catalysed by MDH (Fig. 1). The rate of decrease in absorbance was equivalent to $11 \mathrm{nmol} \mathrm{NADH}$ oxidized/min $\mathrm{x} \mathrm{mg} \mathrm{mito-}$ chondrial protein (Fig. 2Ba). It must, however, be emphasized that this is a considerable overestimate of the true activity of the shuttle as demonstrated by experiments using aminooxyacetate (AOA), a potent transaminase inhibitor which can enter mitochondria (29). First, we confirmed that loading with AOA resulted in essentially complete inhibition of mitochondrial AAT activity (Fig. 2B, inset). When aspartate and $\alpha$-oxoglutarate, the transaminase substrate pair, were added to control mitochondria a fast decrease in the fluorescence of the intramitochondrial pyridine nucleotides occurred as a result of formation of OAA inside mitochondria via mitochondrial AAT and to its reduction to malate via malate dehydrogenase (24). When mitochondria loaded with AOA were used, no change in fluorescence was found, indicating complete inhibition of mitochondrial AAT. Given the mode of operation of the MAL/ASP shuttle (Fig. 1), it is clear that the shuttle should not occur in AOA-loaded mitochondria. However, when such mitochondria were used, the rate of NADH oxidation only decreased by approximately $40 \%$ to $6.5 \mathrm{nmol}$ $\mathrm{NADH}$ oxidized/min $\mathrm{x} \mathrm{mg}$ mitochondrial protein, in agreement with Barron et al (11) (Fig. 2Bb). This residual activity cannot be due to the MAL/ASP shuttle operation, which requires transamination in the mitochondrial matrix, and the true shuttle activity must be represented by the difference between the values in Fig. $2 \mathrm{Ba}$ and b, i.e. $4.5 \mathrm{nmol} \mathrm{NADH}$ oxidized/min $\mathrm{x}$ $\mathrm{mg}$ mitochondrial protein (see below).

One possible explanation for the residual activity in Fig $2 \mathrm{Bb}$ is that a malate/oxaloacetate shuttle exists; this would be consistent with the permeability of heart mitochondria to oxaloacetate (19). Direct evidence for this hypothesis has been obtained (Fig. 2C). When mitochondria were incubated with malate dehydrogenase (MDH, 0.5 e.u.) and NADH alone, i.e. the malate detecting system (MAL D.S.), no change in absorption occurred showing that the OAA concentration in the extramitochondrial phase was negligible. Externally added $1 \mathrm{mM}$ malate (MAL) caused oxidation of NADH at a rate of approximately $6.8 \mathrm{nmol} / \mathrm{min} \mathrm{x} \mathrm{mg}$ protein, indicative of the appearance of OAA outside the mitochondria. In a control experiment, the occurrence of OAA outside mitochondria was confirmed in the supernatants of mitochondria previously added with malate alone. This was performed using both citrate synthase and malate dehydrogenase assays $(30,31)$. In agreement with a previously published study (28), NADH oxidation was strongly inhibited $(\sim 85 \%)$ by $10 \mathrm{mM}$ phenylsuccinate. As in that previous study, a possible explanation of these findings is the following (Fig. 1): MAL can enter mitochondria in exchange with endogenous phosphate or dicarboxylates (29); once inside the matrix MAL is oxidised, by mitochondrial MDH, to OAA which in turn can exit in a manner sensitive to phenylsuccinate. Once outside, it is reduced by $\mathrm{NADH}$ in the presence of $\mathrm{MDH}$ in a reconstructed MAL/ OAA shuttle.

In the light of these findings which show that the activity of the MAL/ASP shuttle, as measured in Fig. 2Ba and previously by Rupert et al (10), is overestimated, being dependent on the efflux of both aspartate and OAA, we developed a new procedure to continuously monitor MAL/ASP shuttle activity (Materials and methods). In this case, the aspartate detecting system (ASP D.S.) was added to the mitochondrial suspension in the sample cuvette, whereas ASP D.S. without AAT was added in the reference cuvette. Then, malate plus glutamate ( $1 \mathrm{mM}$ each) were added simultaneously to both the sample and reference cuvette and the oxidation of NADH was recorded. The true rate of MAL/ASP shuttle was found to be $4.3 \mathrm{nmol} \mathrm{NAD}(\mathrm{P}) \mathrm{H}$ oxidized/min $\mathrm{x}$ mg mitochondrial protein (Fig. 2Da). Consistently, when using AOA-loaded mitochondria, the activity of the shuttle was effectively abolished (Fig. 2Db). As expected, the measured true activity of the MAL/ASP shuttle was very close to the difference between the activities in Fig. 2Ba and b.

The same experiments were carried out with $\mathrm{WKY}_{24}-\mathrm{M}$ and $\mathrm{SHR}_{5}-\mathrm{M}$ and $\mathrm{SHR}_{24}-\mathrm{M}$ with the substrates starting NADH oxidation added at $1 \mathrm{mM}$ concentration (experimental traces not shown here). The rates of NADH oxidation, expressed as nmol NADH oxidized/min x mg mitochondrial protein, were 4.1, 1.8 and 5.2 for the $\alpha \mathrm{GP}$ shuttle; 4.3, 2.9 and 6.2 for the MAL/ASP shuttle; and 11.1, 19.2 and 20.1 for the MAL/ OAA shuttle.

The contribution of the MAL/OAA shuttle to oxidation of NADH is higher than those of the MAL/ASP and $\alpha G P$ shuttles. The dependence of the rate of decrease in absorbance of NADH, i.e. the rate of the in vitro reconstructed shuttles, measured as 

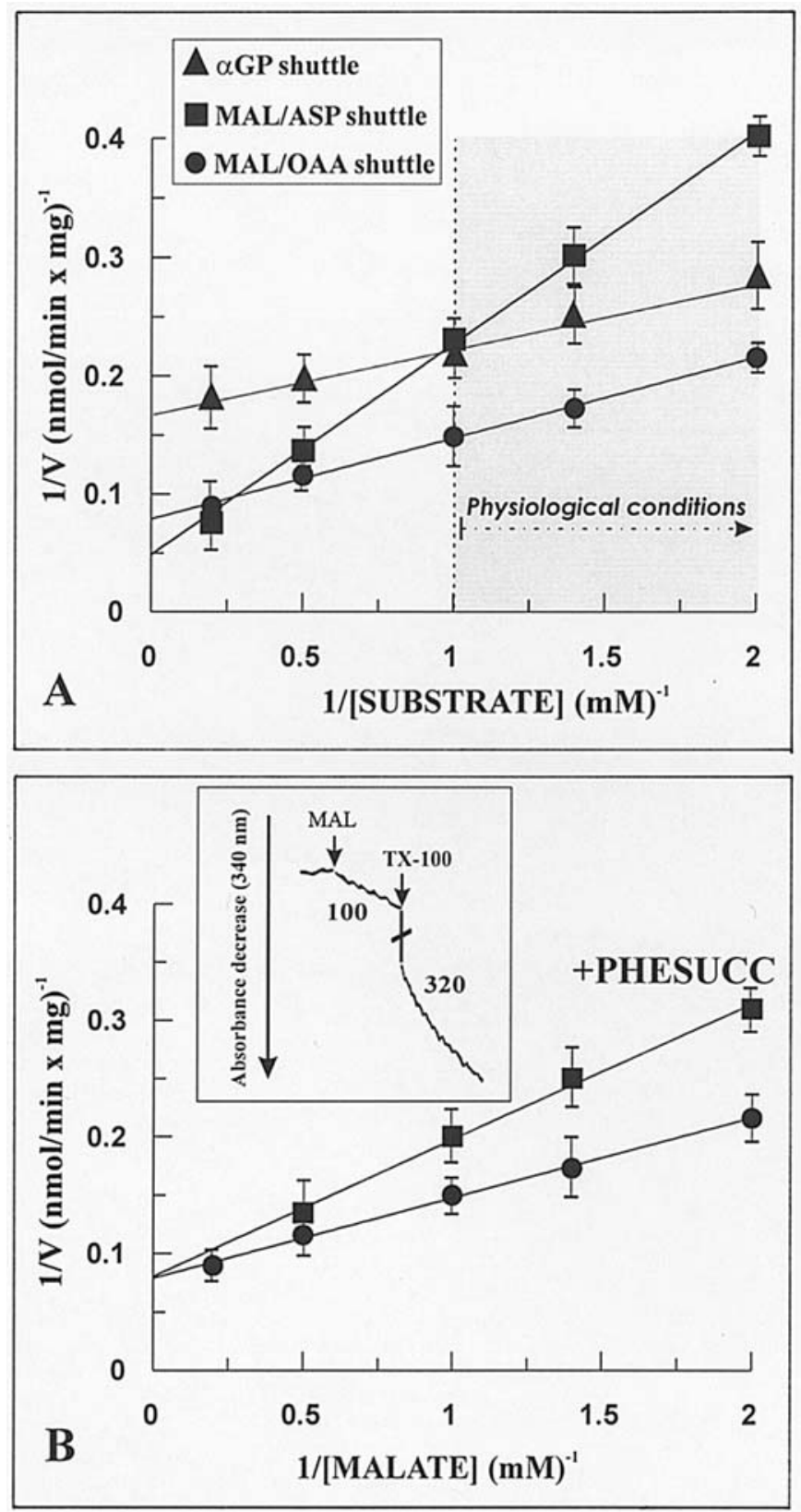

Figure 3. (A) Kinetic analysis of the $\alpha \mathrm{GP}, \mathrm{MAL} / \mathrm{ASP}$ and MAL/OAA shuttles and $(\mathrm{B})$ nature of inhibition by phenylsuccinate of the MAL/OAA shuttle reconstructed by using $\mathrm{WKY}_{5}-\mathrm{M}$. (A) $\mathrm{WKY}_{5}$-mitochondria $(0.5 \mathrm{mg}$ protein) were incubated at $25^{\circ} \mathrm{C}$ in $2.0 \mathrm{ml}$ of standard medium in the presence of NADH $(0.2 \mathrm{mM})$ plus either $\alpha$-glycerol-3-phosphate dehydrogenase ( $\alpha \mathrm{GPDH}, 1$ e.u.) (in the case of $\alpha \mathrm{GP}$ shuttle reconstruction), malate dehydrogenase (MDH, 2 e.u.) (in the case of MAL/OAA shuttle reconstruction) or aspartate amino-transferase (AAT, 1 e.u.) plus MDH (2 e.u.), the substrate pair, $\alpha$-oxoglutarate $(\alpha \mathrm{OG}, 0.1 \mathrm{mM})$ and glutamate (GLU, $1 \mathrm{mM}$ ) (in the case of MAL/ASP shuttle reconstruction) (Materials and methods). The exchange reactions were started by adding $\alpha \mathrm{GP}$ or MAL at the indicated concentrations. $1 \mathrm{mM}$ represents the maximum concentration realizable in cells under physiological conditions. The rate $(\mathrm{v})$, measured as the change in absorbance monitored at $340 \mathrm{~nm}$, is expressed as nmol NADH oxidised/min $\mathrm{x}$ mg mitochondrial protein. (B) The MAL/OAA shuttle reconstruction experiment was performed as described in Figs. 2 and 3A. Malate was added at the indicated concentrations in the absence $(\bullet)$ or presence ( $\boldsymbol{a})$ of phenylsuccinate (PHESUCC, $10 \mathrm{mM}$ ). The rate (v) is expressed as nmol $\mathrm{NADH}$ oxidised/min $\mathrm{x} \mathrm{mg} \mathrm{mitochondrial} \mathrm{protein.} \mathrm{All} \mathrm{assays} \mathrm{were} \mathrm{run} \mathrm{four}$ times and mean \pm SD was reported. The inset shows the TX-100 experiment: TX-100 addition (0.2\%) follows $1 \mathrm{mM}$ MAL addition. Experimental condition as in Fig. 2C, except that mitochondria were incubated with $0.2 \mathrm{mM}$ NADPH (Materials and methods). Numbers along the curves are rates of pyridine nucleotide oxidation expressed as a percentage of control (considered as $100 \%)$, i.e. in the absence of TX-100.
Table II. Kinetic parameters of $\alpha$ GP/DHAP, MAL/ASP and MAL/OAA shuttles in SHR and WKY at 5 and 24 weeks of age and the respective age-matched controls.

$\begin{array}{ccc}\alpha \mathrm{GP} & \mathrm{K}_{\mathrm{m}} \pm \mathrm{SD}(\mathrm{mM}) & \mathrm{V}_{\text {max }} \pm \mathrm{SD} \\ & (\mathrm{nmol} / \mathrm{min} \times \mathrm{mg} \text { protein })\end{array}$

\begin{tabular}{lll}
\hline $\mathrm{SHR}_{5}$ & $0.54 \pm 0.04^{\mathrm{f}}$ & $5.8 \pm 0.29$ \\
$\mathrm{WKY}_{5}$ & $0.52 \pm 0.02^{\mathrm{e}}$ & $6.0 \pm 0.23$ \\
$\mathrm{SHR}_{24}$ & $0.73 \pm 0.04$ & $6.3 \pm 0.23$ \\
$\mathrm{WKY}_{24}$ & $0.77 \pm 0.03$ & $6.0 \pm 0.19$
\end{tabular}

\begin{tabular}{lcc}
\hline MAL/ASP & $\mathrm{K}_{\mathrm{m}} \pm \mathrm{SD}(\mathrm{mM})$ & $\mathrm{V}_{\max } \pm \mathrm{SD}$ \\
$(\mathrm{nmol} / \mathrm{min} \times \mathrm{mg}$ protein $)$
\end{tabular}

\begin{tabular}{lll}
\hline $\mathrm{SHR}_{5}$ & $3.5 \pm 0.8^{\mathrm{e}}$ & $16.8 \pm 0.5^{\mathrm{f}}$ \\
$\mathrm{WKY}_{5}$ & $2.9 \pm 0.7$ & $16.8 \pm 0.3^{\mathrm{f}}$ \\
$\mathrm{SHR}_{24}$ & $1.7 \pm 0.15^{\mathrm{c}}$ & $15.8 \pm 0.6$ \\
$\mathrm{WKY}_{24}$ & $2.6 \pm 0.35$ & $16.0 \pm 0.4$
\end{tabular}

MAL/OAA $\quad \mathrm{K}_{\mathrm{m}} \pm \mathrm{SD}(\mathrm{mM}) \quad \mathrm{V}_{\max } \pm \mathrm{SD}$

(nmol/min $\mathrm{x}$ mg protein)

\begin{tabular}{lcl}
\hline $\mathrm{SHR}_{5}$ & $0.57 \pm 0.05^{\mathrm{a}, \mathrm{d}}$ & $30.0 \pm 0.6^{\mathrm{a}, \mathrm{e}}$ \\
$\mathrm{WKY}_{5}$ & $1.0 \pm 0.3$ & $12.4 \pm 0.3^{\mathrm{e}}$ \\
$\mathrm{SHR}_{24}$ & $1.0 \pm 0.3$ & $40.0 \pm 1.3^{\mathrm{a}}$ \\
$\mathrm{WKY}_{24}$ & $1.0 \pm 0.2$ & $20.3 \pm 0.6$ \\
\hline
\end{tabular}

Mitochondria ( $0.5 \mathrm{mg}$ protein) from either 5- or 24-week old WKY or age-matched $\mathrm{SH}$ rats were suspended at $25^{\circ} \mathrm{C}$ in $2.0 \mathrm{ml}$ of standard medium. Experimental conditions as in Fig. 2. The rate of the shuttle was measured following addition of $1 \mathrm{mM} \alpha \mathrm{GP}$ (for $\alpha \mathrm{GP}$ shuttle) or $1 \mathrm{mM}$ MAL (for both MAL/ASP and MAL/OAA shuttle) and expressed as nmoles NADH oxidized/min $\mathrm{x}$ mg protein. The values of $\mathrm{K}_{\mathrm{m}}$ and $\mathrm{V}_{\max }$ are means $\pm \mathrm{SD}$ of five experiments performed in duplicate. ${ }^{\mathrm{a}} \mathrm{P}<0.001,{ }^{\mathrm{b}} \mathrm{P}<0.01,{ }^{\mathrm{C}} \mathrm{P}<0.05$, when $\mathrm{SHR}_{5}$ or $\mathrm{SHR}_{24}$ vs same-aged WKY; ${ }^{\mathrm{d}} \mathrm{P}<0.001,{ }^{\mathrm{e}} \mathrm{P}<0.01,{ }^{\mathrm{f}} \mathrm{P}<0.05$, when $\mathrm{WKY}_{5}$ or $\mathrm{SHR}_{5}$ vs the respective 24-week-old animals. The lack of asterisk/s indicate no statistically significant difference.

in Fig. 2, was investigated as a function of increasing concentrations of either $\alpha \mathrm{GP}$ or MAL. Hyperbolic reaction kinetics were found for the $\alpha \mathrm{GP}, \mathrm{MAL} / \mathrm{ASP}$ and MAL/OAA shuttles, and Fig. 3A shows a typical double reciprocal plot for data obtained with $\mathrm{WKY}_{5}-\mathrm{M} . \mathrm{K}_{\mathrm{m}}$ values, i.e. the substrate concentration which gives half the maximum rate $\left(\mathrm{V}_{\max }\right)$, and $\mathrm{V}_{\max }$ values were determined in several experiments carried out with different mitochondrial preparations. The values obtained with $\mathrm{SHR}_{5}-\mathrm{M}, \mathrm{SHR}_{24}-\mathrm{M}, \mathrm{WKY}_{5}-\mathrm{M}$ and $\mathrm{WKY}_{24}-\mathrm{M}$ are reported in Table II along with statistical analysis carried out by an ANOVA test. In five experiments, the $\mathrm{V}_{\max }$ values of the MAL/ASP shuttle as measured in $\mathrm{SHR}_{5}$ vs $\mathrm{SHR}_{24}$ differed significantly both from one another and from the same-age WHY samples $(\mathrm{P}<0.05)$. In contrast, no statistically significant differences were found for $\mathrm{V}_{\max }$ values of the $\alpha \mathrm{GP}$ shuttle independently of the age and hypertrophy/hypertension states. 

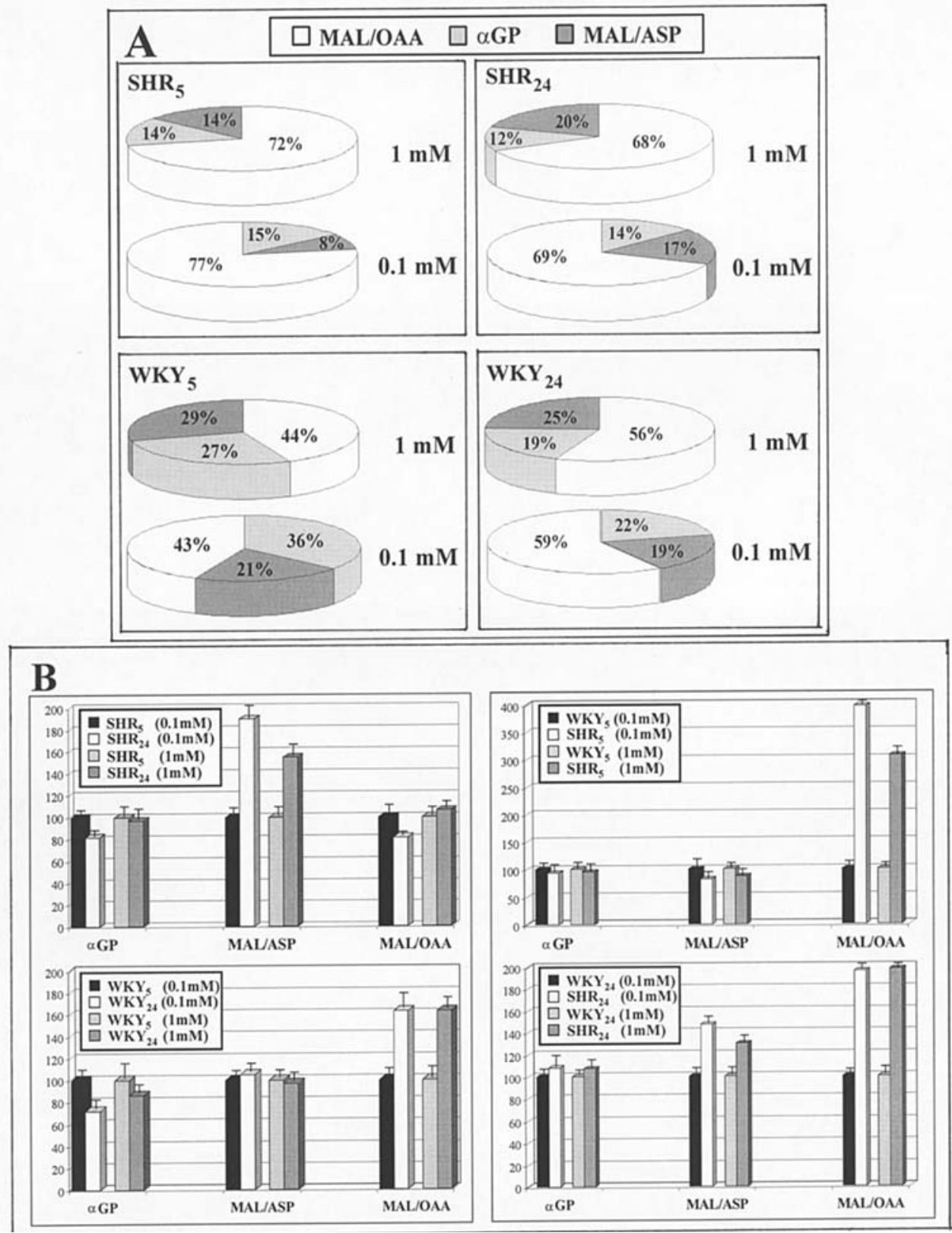

Figure 4. The percentage efficiency of $\alpha \mathrm{GP}, \mathrm{MAL} / \mathrm{ASP}$ and MAL/OAA shuttles. (A) The percentage efficiency of each shuttle in both strains of rats at 5 and 24 weeks. The rates of the shuttles at 0.1 or $1 \mathrm{mM}$ of either malate or $\alpha \mathrm{GP}$ were calculated from the values in Table II. The \% efficiency of each shuttle (X) derives from the following formula:

$$
\% \mathrm{X} \text {-shuttle }=\frac{100 \mathrm{v} \text { (rate of the } \mathrm{X} \text {-shuttle at } 1 \mathrm{mM} \text { substrate })}{(\text { sum of each shuttle rate at } 1 \mathrm{mM} \text { substrate })}
$$

(B) The shuttle efficiency analyzed by dependence on either age for both SHR and WHY rats (left) (100 indicate the value rate at 5 weeks of age of each strain), or hypertrophy/hypertension state (right) (100 indicate the value rate at 5 and 24 weeks of age of WKY rats), with substrates at 0.1 and 1 mM.

The $K_{m}$ values for the MAL/ASP shuttle were found to change significantly in $\mathrm{SHR}_{5}$ vs $\mathrm{SHR}_{24}(\mathrm{P}<0.01)$ and $\mathrm{SHR}_{24}$ vs WKY ${ }_{24}$ $(\mathrm{P}<0.05)$ but no significant change was found when comparing $\mathrm{WKY}_{5} \mathrm{vs}^{\mathrm{WKY}}{ }_{24}$ and $\mathrm{SHR}_{5}$ vs WKY $\mathrm{W}_{5}$. Statistically different $\mathrm{K}_{\mathrm{m}}$ values for the $\alpha \mathrm{GP}$ shuttle were found in $\mathrm{SHR}_{5}$ and $\mathrm{WKY}_{5}$ vs the respective 24 -week old rats $(\mathrm{P}<0.01)$. No statistically significant differences were found in $\mathrm{SHR}_{5}$ and $\mathrm{SHR}_{24}$ vs the respective age-matched normotensive rats.

The $\mathrm{K}_{\mathrm{m}}$ and $\mathrm{V}_{\max }$ values calculated for the MAL/OAA shuttle were found to change in the investigated cases. The $\mathrm{V}_{\max }$ values were found to change significantly with age $\left(\mathrm{WKY}_{5}\right.$ and $\mathrm{SHR}_{5}$ vs the respective 24 -week old rats, $\mathrm{P}<0.01$ ) as well as with hypertrophy/hypertension state $\left(\mathrm{SHR}_{5}\right.$ and $\mathrm{SHR}_{24}$ vs the respective normotensive rats, $\mathrm{P}<0.001)$. Interestingly $\mathrm{K}_{\mathrm{m}}$ values were found to increase significantly both with the age $(\mathrm{P}<0.001)$ and with the progression from the hypertrophy to the hypertrophy/hypertension state $(\mathrm{P}<0.001)$ in SHR, but not with the age of normotensive rats.

The rate of the MAL/OAA shuttle depends on the rate of carrier-mediated substrate transport in both $S H R-M$ and $W H Y-M$. Since the MDH activity added in the malate detecting 
system exceeds the rate of the substrate appearance (Materials and methods), and since malate efflux is a process in which both transport across the mitochondrial membrane and the reaction catalyzed by intramitochondrial dehydrogenase are involved, we checked whether the measured rate of NADH oxidation mirrored the transport process across the mitochondrial membrane. To achieve this, we used the same method as Passarella et al (13); instead of NADH, NADPH was added to $\mathrm{WKY}_{5}-\mathrm{M}$ (Fig. 3B, inset); this substance can be oxidized by the enzyme/s of the detecting systems but not by Complex I of the respiratory chain. After addition of MAL, the detergent, TX-100 (0.2\%), which dissolves mitochondrial membranes, was added. In all cases, the rate of oxidation of NADPH increased dramatically showing that metabolite transport across the mitochondrial inner membrane was the rate limiting step of the measured processes.

Phenylsuccinate, a non-penetrant inhibitor of a variety of mitochondrial carriers, inhibited the malate/oxaloacetate exchange in a competitive manner both in the hypertrophy and in the hypertrophy/hypertension state as well as in the respective controls, i.e. $\mathrm{WKY}_{5}$ (shown as an example in Fig. 3B) and $\mathrm{WKY}_{24}$. The $\mathrm{K}_{\mathrm{i}}$ values, which are indicative of inhibitor-carrier interaction, differed from one another as judged by statistical analysis $(\mathrm{P}<0.05): 1.7 \pm 0.17$ and $2.9 \pm 0.22 \mathrm{mM}$ in $\mathrm{SHR}_{5}$ and $\mathrm{WKY}_{5}$ respectively and $2.1 \pm 0.24$ and $2.8 \pm 0.21 \mathrm{mM}$ in $\mathrm{SHR}_{24}$ and $\mathrm{WKY}_{24}$, respectively.

The rates of the shuttles at 0.1 and $1 \mathrm{mM}$, perhaps maximum malate and $\alpha \mathrm{GP}$ concentration in the cytosol, were calculated from the values in Table II. The results are presented in terms of percentage efficiency of each shuttle in both strains of rats at 5 and 24 weeks (Fig. 4A). In all the cases, the MAL/ OAA shuttle made the greatest contribution to oxidation of NADH (43-77\%), followed by the MAL/ASP shuttle, which was more active than the $\alpha \mathrm{GP}$ shuttle (except for $\mathrm{SHR}_{5}$ where the activity was equal). In Fig. 4B, modifications in shuttle efficiency were analyzed by dependence on age for both SHR and WHY rats, again with substrates at 0.1 and $1 \mathrm{mM}$. A significant increase with age $(\sim 50 \%)$ was found in the activity of the MAL/ASP shuttle in SHR whereas, in normotensive rats, the activity of the MAL/OAA shuttle increased ( $65 \%)$. On the other hand, when normotensive and hypertrophied mitochondria were compared, a huge increase in activity of the MAL/OAA shuttle (200\%) was found. This increase was still apparent in the hypertrophic-hypertensive state, but to a lesser extent.

\section{Discussion}

A change in energy metabolism occurs in the hypertrophied heart, with a decrease in fatty acid oxidation and an increase in glycolytic flux (32). Since left ventricular hypertrophy (LVH) favours the occurrence of arrhythmias and failure and is an independent risk factor for cardiovascular morbidity and mortality, the detection of the modifications in the energy production pathways could be crucial to elucidate how arrhythmias and failure occur and perhaps prevent cardiac dysfunction and transition to overt failure. It has been reported that the glycolytic rate, which is $0.5-1.5 \mu \mathrm{mol}$ glucose oxidised/ $\mathrm{g}$ dry weight in normotensive heart, increases up to 1.5-4 in hypertrophy $(33,34)$. Given that an increase in glycolytic flux results in an increase in NADH production in the cytosol, we have investigated the shuttle mechanisms which allow for the oxidation of cytosolic NADH in the heart. We show here, for the first time, the occurrence of a MAL/OAA shuttle in the left heart ventricle, and demonstrate that it works to transport reducing equivalents from the extramitochondrial phase into the matrix with a higher efficiency than those of the previously reported $\alpha \mathrm{GP}$ and MAL/ASP shuttles. An important consequence of the occurrence of the MAL/OAA shuttle is that previous measurements of the activity of the MAL/ASP shuttle $(10,11,28)$ are seriously overestimated because the contribution of effluxed OAA to the oxidation of external $\mathrm{NADH}$ has not been taken in account. We report the true rate of the MAL/ASP shuttle and show that it makes a minor contribution to oxidation of external NADH. Moreover, we show that the rate of the overall process of NADH oxidation via the MAL/OAA shuttle, as reconstructed in vitro, depends on the rate of metabolite transport across the mitochondrial membrane, probably by MAL/OAA antiport (12). Thus, we confirm that, in the heart, as in the brain, kidney, liver (13-15), plant mitochondria (16) and microorganisms (17), the MAL/ OAA shuttle plays a major role in oxidizing NADH.

When considering the occurrence in vivo of processes shown to occur in vitro, the physiological substrate concentration should be considered. In this case, even though the malate concentration in the heart is between 100 and $500 \mathrm{nmols} / \mathrm{g}$ wet (35), the division between cytosol and mitochondria remains difficult to determine with certainty due to reaching an equilibrium in the time necessary to isolate mitochondria.

However, we can assume that malate concentration is approximately $0.1 \mathrm{mM}$, as reported by Jungling et al (36). We also show that the activity of the MAL/OAA shuttle is dependent on both age and hypertrophy/hypertension progression (Table II and Fig. 4). The MAL/OAA shuttle is more active in SHR than in WKY rats by approximately $140 \%$ and $100 \%$ at 5 and 24 weeks of age, respectively, as expected since aerobic glycolysis increases in hypertrophy and hypertrophy/hypertension $(29,34)$. Interestingly, in both SHR and WKY, an increase in the shuttle activity by approximately $30 \%$ and $60 \%$, respectively, occurs at 24 weeks compared with 5 weeks. It is likely that the increases are higher at physiological malate concentrations. If this concentration is approximately 0.1-1.0 mM (36) and remains constant under hypertrophy/hypertension conditions, the actual rate of the MAL/OAA shuttle should be approximately 4.5-19 and 1.2-6 in SHR and WKY rat mitochondria at 5 weeks of age but approximately 3.6-20 and 1.8-10 in mitochondria from 24week-old SHR and WKY rats respectively. This suggests that in SHR MAL/OAA shuttle efficiency is significantly increased with LVH progression; the activity of the shuttle is similar in 5- and 24-week-old SHR, which exhibit similar values of LVMI but different BP values. Contrarily to SHR, MAL/OAA shuttle increases with ageing in WKY. Nonetheless, in the light of the low $K_{m}$ value for malate $(0.57 \pm 0.05$ and $1.0 \pm 0.3 \mathrm{mM})$ in SHR and WKY at 5 and 24 weeks of age, and of the intracellular concentration (0.1-1.0 mM range) (36), we conclude that this shuttle operates at a rate considerably below the $\mathrm{V}_{\max }$ in both cases. Thus, any increase in NADH formation via glycolysis will result in an increase in the rate of malate 
formation and, finally, in shuttle activity. Such a hypertrophy condition results in modification of the mitochondrial translocator involved in the shuttle, suggested by the different $\mathrm{V}_{\max }$ values and confirmed by the different $\mathrm{K}_{\mathrm{i}}$ values for phenylsuccinate. As expected (32), phenylsuccinate inhibits malate/OAA exchange in a competitive manner.

Neither the MAL/ASP (as properly measured) nor the $\alpha$ GP shuttles are significantly affected in hypertrophied hearts. This is in apparent agreement with Rupert et al (10); however, one could argue that measurements of NADH oxidation via the MAL/ASP shuttle include the MAL/OAA shuttle and, thus, an increase in NADH oxidation should have been found by them. It should be noticed, however, that, in $(10,11,28)$ the MAL/ASP shuttle activity was investigated at $37^{\circ} \mathrm{C}$ under conditions at which most oxidation of NADH would occur spontaneously due to damage of the mitochondrial membrane, resulting in free access of NADH to Complex I. Consequently, any modification in shuttle activity may have been masked. Interestingly, the rate of NADH oxidation via shuttles in the present study is consistent with the reported rate of glucose oxidation to water and carbon dioxide (37). By assuming that $1 \mathrm{~kg}$ of dry weight corresponds to $1 \mathrm{mg}$ of mitochondrial protein, and that glucose oxidation occurs at a rate of approximately $1 \mathrm{nmol} / \mathrm{min} \mathrm{x}$ mg mitochondrial protein, since $\mathrm{H}_{2} \mathrm{O}$ formation derives from the oxidation of $20 \mathrm{NADH}$ and $2 \mathrm{FADH}_{2}$, the total shuttle capacity to oxidize cytosolic NADH appears to be consistent with the reported results. Since electron flow in the respiratory chain and oxidative phosphorylation could take place at a rate much higher than that of NADH oxidation via shuttles, we propose that the glucose oxidation rate depends essentially on the rate of the mitochondrial shuttles and on the rate by which pyruvate, formed in glycolysis, enters mitochondria.

How these results are consistent with the reported enhanced flavin-linked mitochondrial $\alpha \mathrm{GP}$ dehydrogenase activity in adult rats (38) or with the increase in both expression of the mRNA and enzymatic activity of MDH and AAT $(12,39)$ remains to be established. We could speculate that such an increase accompanies the increase in the rate of metabolite transport, which could be the rate limiting step in vivo, as in vitro. On the other hand, because the glutamate/aspartate antiporter is unidirectional and electrogenic, the malate/ aspartate shuttle function might also be limited by overall mitochondrial energetics $(40,41)$.

Whether the changes in NADH reoxidation systems reported here mirror the physiological situation remains a matter of speculation. Indeed, at present, the mechanism by which the reported changes take place is rather obscure; increase in energy demand, related to the development of LVH in SHR, might be seen as the main cause of metabolic changes deriving directly from modifications of certain proteins involved in energy metabolism. Such a putative modification could concern the protein molecule per se and/or the membrane environment. Research to elucidate this point is currently in progress in which gene expression and changes in the protein conformation and lipid environment (42) are under investigation, together with studies of the L-lactate/pyruvate shuttle (43).

The results of the present study, showing the activation of the MAL/OAA shuttle in SHR before the development of hypertension, suggest that this change could be an early marker of LVH, in which 'fuel shift' from fatty acid B-oxidation toward glucose oxidation was previously documented in animal models $(44,45)$ and, more recently, in humans $(39,46)$. Because no further enhancement was found in SHR with development of hypertension, activation of the MAL/OAA shuttle could not be contended as a key mechanism involved in the transition of $\mathrm{LVH}$ in heart failure. $\mathrm{LVH}$, in fact, predisposes the heart to failure and, while metabolic and gene regulatory changes developing in LVH are considered adaptive events, modifications occurring in the failing heart are interpreted as maladaptive because of insufficient ATP production and decreased pump function. Whether derangement in mitochondria energy metabolism could induce heart failure is an intriguing possibility and appropriately designed studies are needed in order to clarify this specific issue.

\section{Acknowledgements}

The authors thank Professor Shawn Doonan for his critical reading of the manuscript and Mr. Vito Giannoccaro for his skilful technical assistance. This study was financed by a grant from MIUR Cluster 03 to E.M.

\section{References}

1. Ingwall JS and Weiss RG: Is the failing heart energy starved? On using chemical energy to support cardiac function. Circ Res 95: 135-145, 2004.

2. Carvajal K and Moreno-Sanchez R: Heart metabolic disturbances in cardiovascular diseases. Arch Med Res 34: 89-99, 2003.

3. Jeffrey FM, Diczku V, Sherry AD and Malloy CR: Substrate selection in the isolated working rat heart: effects of reperfusion, afterload and concentration. Basic Res Cardiol 90: 388-396, 1995.

4. Scheuer J: Fueling the hypertrophied heart. Hypertension 44: 623-624, 2004.

5. El Alaoui-Talibi Z, Landormy S, Loireau A and Moravec J: Fatty acid oxidation and mechanical performance of volumeoverloaded rat hearts. Am J Physiol 262: H1068-H1074, 1992.

6. El Alaoui-Talibi Z, Guendouz A, Moravec M and Moravec J: Control of oxidative metabolism in volume-overloaded rat hearts: effect of propionyl-L-carnitine. Am J Physiol 272: H1615-H1624, 1997.

7. Allard MF: Energy substrate metabolism in cardiac hypertrophy. Curr Hypertens Rep 6: 430-435, 2004.

8. Wambolt RB, Henning SL, English DR, Dyachkova Y, Lopaschuk GD and Allard MF: Glucose utilization and glycogen turnover are accelerated in hypertrophied rat hearts during severe low-flow ischemia. J Mol Cell Cardiol 31: 493-502, 1999.

9. Liao R, Jain M, Cui L, D'Agostino J, Aiello F, Luptak I, Ngoy S, Mortensen RM and Tian R: Cardiac-specific overexpression of GLUT1 prevents the development of heart failure attributable to pressure overload in mice. Circulation 106: 2125-2131, 2002.

10. Rupert BE, Segar JL, Schutte BC and Scholz TD: Metabolic adaptation of the hypertrophied heart: role of the malate/aspartate and alpha-glycerophosphate shuttles. J Mol Cell Cardiol 32: 2287-2297, 2000.

11. Barron JT, Gu L and Parrillo JE: Malate-aspartate shuttle, cytoplasmic NADH redox potential, and energetics in vascular smooth muscle. J Mol Cell Cardiol 30: 1571-1579, 1998.

12. Passarella S, Palmieri F and Quagliariello E: The transport of oxaloacetate in rat heart mitochondria. FEBS Lett 90: 61-64, 1978.

13. Passarella S, Barile M, Atlante A and Quagliariello E: Oxaloacetate uptake into rat brain mitochondria and reconstruction of the malate/oxaloacetate shuttle. Biochem Biophys Res Commun 119: 1039-1046, 1984.

14. Passarella S, Atlante A and Quagliariello E: Oxaloacetate permeation in rat kidney mitochondria: pyruvate/oxaloacetate and malate/oxaloacetate translocators. Biochem Biophys Res Commun 129: 1-10, 1985. 
15. Atlante A, Passarella S and Quagliariello E: Pyruvate/malate antiporter in rat liver mitochondria. Biochem Biophys Res Commun 182: 931-938, 1992.

16. Pastore D, Di Pede S and Passarella S: Isolated durum wheat and potato cell mitochondria oxidize externally added NADH mostly via the malate/oxaloacetate shuttle with a rate that depends on the carrier-mediated transport. Plant Physiol 133: 2029-2039, 2003.

17. Bakker BM, Overkamp KM, van Maris AJ, Kotter P, Luttik MA, van Dijken JP and Pronk JT: Stoichiometry and compartmentation of NADH metabolism in Saccharomyces cerevisiae. FEMS Microbiol Rev 25: 15-37, 2001.

18. Engelmann GL, Vitullo JC and Gerrity RG: Age-related changes in ploidy levels and biochemical parameters in cardiac myocytes isolated from spontaneously hypertensive rats. Circ Res 58: 137-147, 1986.

19. Passarella S, Palmieri F and Quagliariello E: The transport of oxaloacetate in isolated mitochondria. Arch Biochem Biophys 180: 160-168, 1977.

20. Waddel WJ and Hill C: A simple ultraviolet spectrophotometric method for the determination of protein. J Lab Clin Med 48: 311-314, 1956.

21. Doonan S, Marra E, Passarella S, Saccone C and Quagliariello E: Transport of protein into mitochondria. Int Rev Cytol 91: 141-186, 1984.

22. Dawson AG and Cooney GJ: Reconstruction of the alpha-glycerolphosphate shuttle using rat kidney mitochondria. FEBS Lett 91: 169-172, 1978.

23. Atlante A, Gagliardi S and Passarella S: Fumarate permeation in normal and acidotic rat kidney mitochondria: fumarate/malate and fumarate/aspartate translocators. Biochem Biophys Res Commun 243: 711-718, 1998.

24. Marra E, Doonan S, Saccone C and Quagliariello E: Selective permeability of rat liver mitochondria to purified aspartate aminotransferases in vitro. Biochem J 164: 685-691, 1977.

25. Das AM and Harris DA: Defects in regulation of mitochondrial ATP synthase in cardiomyocytes from spontaneously hypertensive rats. Am J Physiol 259: H1264-H1269, 1990.

26. Engelmann GL, Vitullo JC and Gerrity RG: Morphometric analysis of cardiac hypertrophy during development, maturation and senescence in spontaneously hypertensive rats. Circ Res 60: 487-494, 1987.

27. Klingenberg M: Localization of the glycerol-phosphate dehydrogenase in the outer phase of the mitochondrial inner membrane. Eur J Biochem 13: 247-252, 1970.

28. Scholz TD, Koppenhafer SL, TenEyck CJ and Schutte BC: Ontogeny of malate-aspartate shuttle capacity and gene expression in cardiac mitochondria. Am J Physiol 274: C780-C788, 1998.

29. Rognstad R and Katz J: Gluconeogenesis in the kidney cortex. Effects of D-malate and amino-oxyacetate. Biochem J 116: 483-491, 1970.

30. Hohorst H-J and Reim M: Oxaloacetate. In: Meth Enzymol Anal. Bergmeyer HU (ed). pp335-339, 1963.
31. Morgunov I and Srere P: Interaction between citrate synthase and malate dehydrogenase. J Biol Chem 273: 29540-29544, 1998.

32. Passarella S, Atlante A, Valenti D and De Bari L: The role of mitochondrial transport in energy metabolism. Mitochondrion 2: 319-343, 2003.

33. Szewczyk A, Nalecz MJ, Broger C, Wojtczak L and Azzi A: Purification by affinity chromatography of the dicarboxylate carrier from bovine heart mitochondria. Biochim Biophys Acta 894: 252-260, 1987.

34. Leong HS, Brownsey RW, Kulpa JE and Allard MF: Glycolysis and pyruvate oxidation in cardiac hypertrophy - why so unbalanced? Comp Biochem Physiol A Mol Integr Physiol 135: 499-513, 2003.

35. Panchal AR, Comte B, Huang H, Dudar B, Roth B, Chandler M, Des Rosier C, Brunengraber $\mathrm{H}$ and Stanley WC: Acute hibernation decreases myocardial pyruvate carboxylation and citrate release. Am J Physiol Heart Circ Physiol 281: H1613-H1620, 2001.

36. Jungling E, Timmerman M, Ionescu I, Aretz A, Mertens M, Loken C, Kammermeier $\mathrm{H}$ and Fischer Y: Luminometric measurement of subnanomole amounts of key metabolites in extracts from isolated heart muscle cells. Anal Biochem 239: 41-46, 1996.

37. Leong HS, Grist M, Parsons H, Wambolt RB, Lopaschuk GD, Brownsey R and Allard MF: Accelerated rates of glycolysis in the hypertrophied heart: are they a methodological artifact? Am J Physiol Endocrinol Metab 282: E1039-E1045, 2002.

38. Sambandam N, Lopaschuk GD, Brownsey RW and Allard MF: Energy metabolism in the hypertrophied heart. Heart Fail Rev 7: 161-173, 2002.

39. Atlante A, Abruzzese F, Seccia TM, Vulpis V, Doonan S, Pirrelli A and Marra E: Changes in enzyme levels in hypertensive heart tissue. Biochem Mol Biol Int 37: 983-990, 1995.

40. Scholz TD and Koppenhafer SL: Reducing equivalent shuttles in developing porcine myocardium: enhanced capacity in the newborn heart. Pediatr Res 38: 221-227, 1995.

41. La Noue KF and Tischler ME: Electrogenic characteristics of the mitochondrial glutamate-aspartate antiporter. J Biol Chem 249: 7522-7528, 1974.

42. Paradies G, Ruggiero FM and Dinoi P: Decreased activity of the phosphate carrier and modification of lipids in cardiac mitochondria from senescent rats. Int J Biochem 24: 783-787, 1992.

43. Valenti D, De Bari L, Atlante A and Passarella S: L-Lactate transport into rat heart mitochondria and reconstruction of the L-lactate/pyruvate shuttle. Biochem J 364: 101-104, 2002.

44. Christe ME and Rodgers RL: Altered glucose and fatty acid oxidation in hearts of the spontaneously hypertensive rat. J Mol Cell Cardiol 26: 1371-1375, 1994.

45. Davila-Roman VG, Vedala G, Herrero P, De las Fuentes L, Rogers JG, Kelly DP and Gropler RJ: Altered myocardial fatty acid and glucose metabolism in idiopathic dilated cardiomyopathy. J Am Coll Cardiol 40: 271-277, 2002.

46. Razeghi P, Young ME, Alcorn JL, Moravec CS, Frazier OH and Taegtmeyer H: Metabolic gene expression in fetal and failing human heart. Circulation 104: 2923-2931, 2001. 